\section{Counter-conditioning as an intervention to modify anti-fat attitudes}

\author{
Stuart W. Flint,1 Joanne Hudson,2 \\ David Lavallee ${ }^{3}$
}

1 Academy of Sport and Physical Activity, Health and Wellbeing Research Institute, Sheffield Hallam University; 2Department of Sport and Exercise Science, Aberystwyth University; ${ }^{3}$ School of Sport, University of Stirling, United Kingdom

\section{Abstract}

This study examined the effect of anti-fat attitude counter-conditioning using positive images of obese individuals participants completed implicit and explicit measures of attitudes towards fatness on three occasions: no intervention; following exposure to positive images of obese members of the general public; and to images of obese celebrities. Contrary to expectations, positive images of obese individuals did not result in more positive attitudes towards fatness as expected and, in some cases, indices of these attitudes worsened. Results suggest that attitudes towards obesity and fatness may be somewhat robust and resistant to change, possibly suggesting a central and not peripheral processing route for their formation.

\section{Introduction}

To date there have been limited efforts to reduce implicit or explicit anti-fat attitudes and these have demonstrated varying effectiveness.1,2 Interventions aimed at reducing anti-fat attitudes include modifying existing knowledge and beliefs about the causes and controllability of overweight and obesity, ${ }^{3,4}$ or evoking empathy for these individuals. 5 A number of studies have reported no change in attitude or modest positive effects. ${ }^{6,7}$ Others have produced more promising results, for example, participants receiving information about the uncontrollable causes of obesity reported reduced anti-fat attitudes whereas those receiving information that obesity is caused by controllable factors demonstrated an increase in anti-fat attitudes. ${ }^{8}$ Although interventions to modify anti-fat attitudes have had limited effectiveness, greater success has been observed with attitudes towards other individuals and groups. For instance based on the premise that we associate positive attrib- utes with accomplished, famous individuals, counter-conditioning involving exposure to well known black individuals (i.e., famous black athletes, politicians, actors) has been used to modify racial attitudes. ${ }^{9}$ Compared with exposure to non-racial or pro-white images participants' initial white preference reduced. Importantly, this effect was observed for implicit but not explicit attitudes.

A plethora of research has examined attitude change in relation to media and advertising stimuli and the role of persuasion in shaping existing attitudes and has been more successful.10,11 A common framework for this research, the Elaboration Likelihood Model of persuasion and attitude formation, 12 proposes two routes to explain attitude change. Central route processing involves repeated exposure and direct attention to relevant stimuli producing robust attitudes. In contrast, peripheral route processing is less complex, whereby attitudes are shaped by superficial aspects of a message (e.g., source credibility) resulting in weaker, more malleable attitudes. 12 Consequently, less cognitive effort is required to change attitudes formed via this route. These two routes by which attitudes are formed may therefore account for the difference in effectiveness of attitude interventions.

Two studies have examined the effectiveness of an intervention comprised of counterconditioning and evoking empathy to reduce anti-fat attitudes. The first employed a variety of conditions as part of the intervention: a video of an obese female discussing her experiences of discrimination, written facts related to the relationship between the environment and genetics and obesity and role-play where an obese person's perspective was taken by the participant. ${ }^{13}$ Weise and colleagues reported that beliefs about the controllability of obesity reduced but it was unclear whether counterconditioning or evoking empathy was responsible for this change. However, a recent review of anti-fat intervention studies has questioned these findings, 1 on the basis that initial between group differences at baseline were not accounted for

In the second of these studies ${ }^{6}$ participants were exposed to four 10 minute videos (evoking empathy, control video, counter stereotypical portrayal, stereotypical portrayal). These interventions induced little change in both implicit and explicit attitudes. Indeed a counterintuitive trend was identified by Gapinski and colleagues, where explicit anti-fat attitudes reduced after exposure to the stereotypical portrayal. These findings may have been the result of overexposure to anti-fat portrayals which consequently may have been condemned by participants leading to lower explicit anti-fat attitudes. As a result of the limitations of the above studies, the effectiveness of counter-conditioning as an anti-fat attitude
Correspondence: Stuart Flint, Collegiate Hall, Collegiate Crescent Campus, Sheffield Hallam University, Sheffield, S10 2BP, United Kingdom. Tel. +44.0114.225.5582 - Fax: +44.0114.225.4377 E-mail:s.flint@shu.ac.uk.

Key words: counter-conditioning, attitude strength, obesity.

Contributions: the authors contributed equally.

Conflict of interests: the authors report no conflict of interests.

Received for publication: 21 December 2012. Accepted for publication: 14 January 2013.

This work is licensed under a Creative Commons Attribution NonCommercial 3.0 License (CC BYNC 3.0).

(C) Copyright S.W. Flint et al., 2013

Licensee PAGEPress, Italy

Health Psychology Research 2013; 1:e24

doi:10.4082/hpr.2013.e24

intervention is still unclear and requires further examination. Future research should attend to the limitations of both these studies when examining anti-fat attitude interventions. First, interventions should be examined separately to ensure that different interventions can be clearly delineated unlike in Weise et al. ${ }^{13}$ Second, attitude formation is vulnerable to social desirability influences therefore an individual's attitude is often similar to those of others around them. ${ }^{14}$ Thus, a major limitation of Gapinski et al. ${ }^{6}$ is that participants were exposed to the video conditions and completed study measures in small groups which may have had an effect on participants' responses, leading to a non-significant effect.

Thus negative images of obese individuals can heighten anti-fat attitudes but whether positive images can decrease these attitudes is unknown. The aim of the present study was to examine the effect of a counter-conditioning intervention using positive images of obese members of the general public and celebrities on anti-fat attitudes. Based on extant research ${ }^{9}$ it was hypothesized that both sets of images would result in reductions in anti-fat attitudes, with a greater effect of celebrity images.

\section{Materials and Methods}

\section{Participants}

Twenty eight volunteer participants (17 men, 11 women; aged $18-35$ years, mean age = $22.43, \quad \mathrm{SD}_{ \pm} .59$ years) were recruited. 
Institutional ethics approval was granted and informed consent provided by participants.

\section{Measures}

Participants reported their age, sex, exercise frequency and perceptions of the words fat (Q1: How insulting do you believe the word fat is?) and obese (Q2: How insulting do you believe the word obese is?). Their height and weight were measured by the first author to calculate their Body Mass Index (BMI) using the following equation: $\mathrm{BMI}=$ weight $(\mathrm{kg}) /$ height $\left(\mathrm{m}^{2}\right)$. To respond to $\mathrm{Q} 1$ and $\mathrm{Q} 2$ participants' used a response scale of $0-10$ which was anchored by $0=$ not at all and $10=$ extremely insulting. Participants' also completed online versions of the Attitudes Towards Obese Persons and Beliefs About Obese Persons scales (ATOP, BAOP) 15 that measure positive and negative attitudes towards obese people and perceived controllability of obesity, respectively. The ATOP has 20 items with scores ranging from 0-120 where low scores represent more negative attitudes and the BAOP has 8 items with scores ranging from 0 48 where low scores represent a stronger belief that obesity is controllable. Participants also completed the Anti-Fat Attitudes Scale (AFAS) ${ }^{16}$ that includes 5 items measuring the magnitude of anti-fat attitudes, where scores range from 0 -25 (higher scores represent stronger anti-fat attitudes), the F-Scale (Shortened version of the Fat Phobia scale) ${ }^{17}$ with 14 items measuring the degree to which individuals associate stereotypical characteristics with being fat (responses range from 0-5 where higher scores are indicative of a perception that the characteristics are associated with being fat).

The final computer-based measure was a version of the Implicit Association Test (IAT) ${ }^{18}$ designed to measure implicit attitudes towards fatness and thinness, using stimuli words employed previously. 19 The measure provides an indication of the individual's implicit preference for fatness or thinness as opposed to a direct measure of absolute attitudes. Participants are presented with weight related words and associate these as quickly as possible with one of two pairings: fatpleasant and thin/unpleasant or fat/unpleasant and thin/pleasant. The seven step procedure outlined in prior work was employed, ${ }^{20}$ where participants were required to respond to each of the pairings once familiarised with the test: (1) pleasant or unpleasant; (2) fat or thin; (3) fat/pleasant or thin/unpleasant; (4) fat/pleasant or thin/unpleasant; (5) thin or fat (6) fat/unpleasant or thin/pleasant; (7) fat/unpleasant or thin/pleasant. Only steps 3,4 , 6 and 7 are used to measure respondents' implicit attitudes (see below); the remaining steps are practice stimuli to engage participants with the process. Participants are pre- sented with a word in the middle of the screen and are required to associate that word with either of the grouping categories in the top left or top right of the screen using the E or I keys, respectively (e.g., for happy pleasant is located in the top left and unpleasant in the top right). Response latency to the different pairings is measured in milliseconds, where positive scores represent stronger anti-fat or pro-thin bias (see below for the range of scores included). Response scales for the explicit measures were a six point likert-type scale from +3 to -3 for the ATOP and BAOP (excluding 0 ) and 1-5 for both the AFAS and F-Scale. Higher scores on the AFAS, F-Scale, Q1 and Q2 signify more negative attitudes and lower scores on the ATOP and BAOP. The ATOP (e.g., $\alpha$ coefficient of 0.76$),{ }^{21}$ BAOP ( $\alpha$ coefficient of 0.82$),{ }^{21}$ AFAS $(\alpha=0.80)^{16}$ and the F-Scale $(\alpha=0.87)^{17}$ have all been demonstrated to be reliable.

\section{Procedures}

Using a within-subjects design (i.e., participants acted as their own controls), participants attended the laboratory on three separate occasions on different days. In the initial baseline condition participants completed all measures and received no manipulation. In the two experimental conditions participants were either exposed to a slideshow of overweight and obese members of the general public or celebrities (11 male and 11 female; $81 \%$ white for both conditions), presented in counter-balanced order. All images were non-stereotypical; for example, images of doctors and fitness instructors in the general public condition, and in the celebrity condition the image was accompanied by information detailing the individual's achievements. This information was included to reinforce positive associations with the celebrities, as it has been suggested that this association impacted the reduction of negative racial attitudes. ${ }^{9}$ Thirty images were sampled from the internet and three independent researchers examined the images to ensure they did not promote the stereotypes associated with overweight and obese, as measured in the F-scale.17 After the intervention participants completed the measures described above.

Results from a pilot study ( $\mathrm{n}=5)$ indicated that scores on the BAOP, 15 AFAS 16 and F-Scale ${ }^{17}$ became more positive in response to these stimuli. Verbal feedback suggested that individuals in the public images were perceived as positive and non-stereotypical, whilst celebrity images were recognised as portraying admirable individuals.

\section{Data analysis}

Apart from the IAT, all total scores for each measure were calculated and used in analyses. IAT D scores were calculated as recommen ded, 22 representing the difference between total response latency for the pairings fatpleasant and thin/unpleasant versus fat/unpleasant and thinpleasant to represent implicit attitudes: (1) delete responses greater than $10,000 \mathrm{~m} / \mathrm{sec}(2)$ delete participants' data where more than $10 \%$ of responses have a response latency less than $300 \mathrm{msec}$ (3) compute the inclusive standard deviation for all responses in steps 3 and 6 and similarly in 4 and 7 (4) compute the mean latency for responses in steps $3,4,6$ and 7 (5) compute the main differences (mean step 6 - mean step 3 and mean step 7 - mean step 4) (6) divide each difference score by its associated inclusive standard deviation and (7) calculate the D score as the equal weight mean of the two resulting ratios. Positive scores are indicative of anti-fat attitudes.

Repeated measures one-way ANOVAs with Bonferroni correction for confidence interval adjustment and follow up post-hoc tests with Scheffé correction were used to compare attitudes between conditions. Paired t-tests were conducted to locate between conditions differences indicated by significant overall effects, with alpha set at 0.05 .

\section{Results}

Cronbach's alpha calculated across all conditions was acceptable for all explicit measures $(\mathrm{ATOP}=0.8, \quad \mathrm{BAOP}=0.7, \quad \mathrm{AFAS}=0.7, \quad \mathrm{~F}$ Scale $=0.9$ ). Higher IAT D scores (indicating anti-fat/pro thin bias) were observed in both experimental conditions compared with baseline (Table 1). Despite the difference in IAT D scores, there was no significant difference $[F(2,52)=1.04, P>0.05]$. Attitudes towards obese persons (ATOP) and the magnitude of anti-fat attitudes (AFAS) appeared to worsen in comparison to the baseline when exposed to the public and celebrity conditions; however these differences were non-significant: $F(2$, $52)=0.41, \mathrm{P}>0.05 ; \mathrm{F}(1.5,37.7)=1.2, \mathrm{P}>0.05$ respectively (Table 1). Although non-significant, findings did however suggest that beliefs about the controllability of obesity (BAOP) and associating negative characteristics with fatness (F-Scale) improved after exposure to the public and celebrity images in comparison to baseline: $\mathrm{F}(2,52)=0.14, \mathrm{P}>0.05 ; \mathrm{F}(2,52)=$ $0.52, \mathrm{P}>0.05$ respectively (Table 1 ).

The only significant effect for explicit attitudes indicated that participants perceived $f a t$ differently in the three conditions $[F(2,52)=$ $4.69, \mathrm{P}<0.05]$. Follow-up tests indicated that fat was perceived as more insulting in the celebrity condition compared with baseline conditions $[t(27)=-3.22, \mathrm{P}<0.01]$. 


\section{Discussion}

Contrary to our hypothesis, exposure to positive images of the obese did not result in more positive perceptions of obesity; hence, according to the Elaboration Likelihood Model central and not peripheral processing may be involved in forming attitudes towards the overweight and obese 12 The central route involves complex processing meaning attitudes are less likely to be shaped by underlying messages, and the peripheral route involves less complex processing meaning attitudes are more unstable and thus more likely to be altered by underlying messages. Both implicit and explicit attitudes showed little change, or viewed another way, existing anti-fat attitudes were reinforced, thus endorsing this suggested role of central processing.

Effective attitude shaping is, however, dependent on the strength of the underlying message, which can be increased by length of exposure to scrutinize the persuasive message. ${ }^{7}$ Therefore longer exposure to the counter-conditioning manipulation may have yielded results more in line with expectations. Additionally, motivation is key to attitude change and which of the two routes of processing is employed.12 Participants' motivation to alter their attitudes was not assessed in this study, but may help to explain why no change in attitude was observed. Future research should measure motivation to change anti-fat attitudes and where motivation is low employ a manipulation strategy to modify this, such as highlighting the negative potential impacts of holding these attitudes.

Although counter-conditioning interventions have positively influenced racial attitudes in previous research a similar intervention used here to modify anti-fat attitudes did not produce a positive effect. ${ }^{9}$ The ineffectiveness of this intervention does however reflect the limited success of other interventions aimed at modifying anti-fat attitudes. ${ }^{7}$ Previous work suggests that negative images of obese individuals heighten anti-fat attitudes ${ }^{23}$ but producing the opposite effect with positive images appears to be more difficult. Our results do not inform why this may be the case but future studies could seek to address this apparent contradiction (e.g., by monitoring appraisals of the images presented).

As our results indicate that anti-fat attitudes either did not change or became more negative following counter-conditioning designed to improve these, this may suggest that these attitudes are somewhat robust and resistant to change. In accordance with previous research, ${ }^{23}$ in our sample both implicit and explicit attitudes were overwhelmingly negative. Our results also support previous findings that exposure to images of overweight individ- uals led to increased stigmatization and perceptions of the overweight as lonely, lazy and teased. ${ }^{24}$ Swami et al. ${ }^{24}$ suggest that this is not surprising, as the more discrepant a body size is from the perceived societal ideal of physical attractiveness the more likely stigmatization and stereotyping is to occur.

Following earlier work, ${ }^{9}$ we hypothesized that celebrity images would result in more positive changes in anti-fat attitudes than would images of the general public. This hypothesis was not supported, suggesting perhaps that the association between public status and desired characteristics observed previously does not extend to perceptions of obese individuals. This may illustrate that anti-fat attitudes have a different basis from other attitudes (e.g., racial attitudes). For instance, discrimination laws exist against racism and sexism, and skin colour and race are not within the individual's control. In contrast, no such legislation currently exists in the United Kingdom in relation to obesity, ${ }^{25}$ and this condition is perceived by some as at least under the individual's partial control of the individual. ${ }^{5}$ Hence, it may be that anti-fat attitudes are perceived as more acceptable, making them more resistant to change, irrespective of factors such as status that help to facilitate change in other attitudes. Research that explores this suggestion and the factors that contribute to the development of anti-fat attitudes would therefore seem important.

\section{Limitations}

A limitation of this study that future research should address is the relatively short duration of exposure to the images of overweight and obese populations in comparison to some previous uses of counter-conditioning to modify other attitudes. ${ }^{26}$ Additionally, although celebrities might be perceived positively, this may not be sufficient to endorse a message to an audience, as a celebrity is more likely to have an effect if they are perceived as relevant to the message being conveyed. ${ }^{9}$ Thus, the descriptions used in the celebrity condition may have detracted from the main message of reducing anti-fat attitudes where participants' attention may have been focused on the celebrities' achievements as opposed to their body shape. Final limitations are the lack of follow up and control for the effect of differentiating participant characteristics. However, whilst examining the difference of the intervention between BMI categories may have been useful, previous research (e.g., Puhl \& Brownell ${ }^{21}$ has demonstrated that overweight and obese individuals also report negative attitudes towards obesity, reinforcing the need to intervene with individuals across the body size spectrum.

\section{Future research directions}

There are a number of potential research questions to examine as a result of this study. First, why do individuals develop anti-fat attitudes and why do they appear to be so ingrained? Second, why do anti-fat attitudes appear to worsen through exposure to images of overweight and obese individuals as demonstrated in the present study? Although confirmation is needed, this finding is concerning and requires attention.

Future research should also examine the acceptability of anti-fat attitudes, as this may shed light on why they appear to be resistant to change, as motivation is a key determinant of

Table 1. Means, standard deviation in parentheses and F statistics for explicit and implicit attitudes in relation to baseline, celebrity and general public conditions.

\begin{tabular}{lcccc} 
Measure & Baseline & Celebrity & General public & F (d.f., error d.f) \\
ATOP & 60.93 & 59.11 & 59.32 & 0.41 \\
& $(12.95)$ & $(16.94)$ & $(16.87)$ & $(2,52)$ \\
BAOP & 12.11 & 12.64 & 12.75 & 0.14 \\
& $(4.47)$ & $(5.06)$ & $(7.33)$ & $(2,52)$ \\
\hline AFAS & 17.45 & 17.93 & 17.71 & 1.20 \\
& $(3.43)$ & $(3.43)$ & $(3.40)$ & $(1.5,37.7)$ \\
F-Scale & 4.03 & 3.98 & 3.99 & 0.52 \\
& $(0.47)$ & $(0.50)$ & $(0.47)$ & $(2,52)$ \\
\hline Q1 & 7.54 & 8.07 & 7.75 & 4.69 \\
& $(1.45)$ & $(1.33)$ & $(1.35)$ & $2.52)$ \\
Q2 & 6.50 & 6.64 & 7.11 & $(1.6,42.2)$ \\
& $(2.56)$ & $(2.20)$ & $(2.13)$ & 0.36 \\
IAT D score & 690 & 820 & 770 & $(2,52)$ \\
$(\mathrm{m} /$ sec $)$ & $(540)$ & $(410)$ & $(400)$ & \\
\hline
\end{tabular}

ATOP, Attitudes About Obese Persons Scale; BAOP, Beliefs About Obese Persons Scale; AFAS, Anti-Fat Attitudes Scale; F-Scale, The Fat Phobia Scale short form; IAT: Implicit Attitudes Test modified to assess attitudes towards fatness and thinness; Q1, How insulting do you believe the word fat is?; $\mathrm{Q} 2$, How insulting do you believe the word obese is? 
attitude change. ${ }^{8}$ If anti-fat attitudes are perceived as acceptable, this would also explain why they are reported in explicit measures. Qualitative methods to examine how acceptable anti-fat attitudes are and the complexity of attitude change may shed light on why these attitudes appear to be more resistant to change than attitudes towards other physical characteristics.

Finally, the paucity and effectiveness of attempts to reduce anti-fat attitudes calls for future research into attitude modification interventions. This research should endeavour to overcome the obstacle of robust attitudes by ensuring that interventions allow individuals to diligently consider information included and experience repeated exposure to increase the likelihood of central as opposed to peripheral route processing.

\section{Conclusions}

With the increasing prevalence of obesity in the United Kingdom, ${ }^{27}$ this research is timely and answers the calls for examination of antifat attitude reduction strategies as, to date, these lack inspection. ${ }^{8}$ This research has made an important contribution to this area of enquiry by suggesting the robustness of antifat attitudes. From a theoretical perspective, this research provides evidence that anti-fat attitudes remained resistant to change reflecting those processed through the central route of the Elaboration Likelihood Model. 12

Although this study contributes to the literature examining anti-fat attitude reduction interventions, this research is still in its infancy. Contrary to our expectations, attitudes were not improved but worsened, irrespective of the fact that participants were presented with positive images of obese individuals. Although support for our hypothesis was lacking present findings are all the more interesting and indeed worrying as a result. Our findings raise questions concerning the route by which antifat attitudes are formed, their apparent robustness, and influences on these attitudes (e.g., lack of anti-obesity discrimination legislation and perceived controllability of obesity). These appear worthwhile to address in subsequent studies.

\section{References}

1. Dan elsd ttir S, 0'Brien KS, Ciao A. Antifat prejudice reduction: a review of published studies. Obesity Facts 2010;3:47-58.

2. 0'Brien KS, Hunter JA, Halberstadt J, Anderson J. Body image and explicit and implicit anti-fat attitudes: the mediating role of physical appearance comparison. Body Image 2007;4:249-56.

3. Anesbury T, Tiggemann M. An attempt to reduce negative stereotyping of obesity in children by changing controllability beliefs. Health Educ Res 2000;15:141-52.

4. Diedrichs PC, Barlow FK. How to lose weight bias fast! Evaluating a brief antiweight bias intervention. Brit $\mathrm{J}$ Health Psych 2011;16:846-61.

5. Teachman BA, Gapinski KD, Brownell KD, et al. Demonstrations of implicit anti-fat bias: the impact of providing causal information and evoking empathy. Health Psychol 2003;22:68-78.

6. Gapinski KD, Schwartz MB, Brownell KD. Can television change anti-fat attitudes and behavior? J Appl Biobehav Res 2006;11: 1-28.

7. Hague AL, White AA. Web-based intervention for changing attitudes of obesity among current and future teachers. J Nutr Educ Behav 2005;37:58-66.

8. 0'Brien KS, Puhl RM, Latner JD, et al. Reducing anti-fat prejudice in pre-service health students: a randomised trial. Obesity 2010;10:1-7.

9. Dasgupta A, Greenwald AG. On the malleability of automatic attitudes: combating automatic prejudice with images of admired and disliked individuals. J Pers Soc Psychol 2001;81:800-14.

10. Berry TR, McLeod NC, Pankratow M, Walker J. Effects of biggest loser exercise depictions on exercise-related attitudes. Am J Health Behav 2013;37:96-103.

11. Petty RE, Cacioppo JT, Schumann D. Central and peripheral routes to advertising effectiveness. The moderating role of involvement. J Consum Res 1983;10:13546.

12. Petty RE, Cacioppo JT. The elaboration likelihood model of persuasion. In: Berkowitz L, ed. Advances in social psychology. New York: Academic Press; 1986. pp 124-181.

13. Wiese HJC, Wilson JF, Jones RA, Neises M. Obesity stigma reduction in medical stu- dents. Int J Obesity 1992;16:859-68.

14. Taylor JB. What do attitude scales measure: the problem of social desirability. J Abnorm Soc Psych 1961;62:386-90.

15. Allison DB, Basile VC, Yuker HE. The measurement of attitudes toward and beliefs about obese persons. Int $\mathrm{J}$ Eat Disorders 1991;10:599-607.

16. Morrison TG, O'Connor WE. Psychometric properties of a scale measuring negative attitudes toward overweight individuals. J Soc Psychol 1999;139:752-95.

17. Bacon JG, Scheltema KE, Robinson BE. Fat phobia scale revisited: the short form. Int $\mathrm{J}$ Obesity 2005;25:252-7.

18. Greenwald AG, McGhee DE, Schwartz JLK. Measuring individual differences in implicit cognition: the implicit association test. J Pers Soc Psychol 1998;74:1464-80.

19. Vartanian LR, Herman CP, Polivy J. Implicit and explicit attitudes towards fatness and thinness: the role of the internalization of societal standards. Body Image 2005;2:373-81.

20. Lane KA, Banaji MR, Nosek BA, Greenwalk AG. Understanding and using the Implicit Association Test: IV. In: Wittenbrink B, Schwartz N, eds. Implicit measures of attitudes. New York: The Guilford Press; 2007. pp 59-102.

21. Puhl RM, Brownell KD. Confronting and coping with weight stigma: an investigation of overweight and obese adults. Obesity 2006;14:1802-15.

22. Greenwald AG, Nosek BA, Banaji MR. Understanding and using the Implicit Association Test: an improved scoring algorithm. J Pers Soc Psychol 2003;85:197216.

23. McClure KJ, Puhl RM, Heuer CA. Obesity in the news: do photographic images of obese persons influence antifat attitudes. J Commun 2011;0:1-3.

24. Swami V, Furnham A, Amin R, et al. Lonelier, lazier and teased: the stigmatizing effects of body size. J Soc Psych 2008;148:577-93.

25. The Equality Act. Available from: www.legislation.gov.uk.

26. Litcher JH, Johnson DW. Changes in attitudes towards negroes of white elementary school students after use of multiethnic readers. J Educ Psychol 1969;60:14852.

27. James WPT. WHO recognition of the global obesity epidemic. Int J Obesity 2008;32: S120-6. 